\title{
Emergency Rescue Location Model with Uncertain Rescue Time
}

\author{
Jing Guan \\ College of Science, Civil Aviation University of China, Tianjin 300300, China \\ Correspondence should be addressed to Jing Guan; jguan@cauc.edu.cn \\ Received 8 May 2014; Accepted 14 November 2014; Published 1 December 2014 \\ Academic Editor: Purushothaman Damodaran \\ Copyright (C) 2014 Jing Guan. This is an open access article distributed under the Creative Commons Attribution License, which \\ permits unrestricted use, distribution, and reproduction in any medium, provided the original work is properly cited. \\ In order to model emergency rescue location problem with uncertain rescue time, an uncertain expected cost minimization model \\ is proposed under uncertain environment. For solving this model, we convert the uncertain model to its equivalent deterministic \\ form. Finally, a numerical example has been presented to illustrate the model. The computational results which were solved by the \\ down mountain algorithm are provided to demonstrate the effectiveness of the model.
}

\section{Introduction}

In recent years, all kinds of unexpected disastrous events continue to occur all over the world [1], such as terrorist attacks on the World Trade Center, "SARS," "5.12" Wenchuan earthquake and Yushu earthquake in China, serious droughts in the five provinces of southwest China, and Japan's nuclear leak. These disasters have caused great property loss for people. Therefore, it is essential to research the sudden disaster emergency management and its logistics system.

Emergency rescue center location is a vital problem in the field of emergency logistics management [1], and it is also an important tool to achieve effective rescue resources distribution and scheduling after the disaster. Emergency rescue center location problem is different from normal locationallocation problems. The difference is mainly reflected in the uncertainty of demand for the disaster area and the urgency of the rescue time. Large-scale disaster emergency rescue needs to mobilize large-scale emergency supplies. Effective emergency rescue is very important to reduce casualties. However, in the case of a large disaster, people needs to transport a lot of aid supplies and rescuers to the disaster area in a short time. Because of burst and uncertainty on disaster and demand for emergency supplies, we must make reasonable optimization for emergency location in order to make the entire emergency rescue network work effectively [2].
Scholars have made a lot of research on emergency location problem. Cooper [3] proposed facility locationallocation problem which has been widely used in the areas of emergency location. In the premise of covering all demand point customers, Roth [4] and Toregas and Swaim [5] studied the minimum cost of facilities location of set covering problem; Murali et al. [6] studied emergency logistics facility location problem with capacity constraints and established the maximum covering location model. Murtagh and Niwattisyawong [7] studied facility location-allocation problem with capacity constraints.

Harewood [8] has calculated the emergency rescue probability using queuing theory in rescue cover problem and studied emergency rescue problem with the minimum cost. Brotcorne et al. [9], Goldberg [10], Alsalloum and Rand [11], and Tovia [12] have studied emergency location problem which is subject to the time and cost. In the premise of meeting the urgency of rescue time, Fang and $\mathrm{He}$ [13] proposed the minimum total cost model. Wang and Zhang [14] proposed emergency rescue center location model which was based on the probability of disasters, spread function of disasters, and rescue function and was solved by embedded heuristic genetic algorithm. Ma et al. [15] researched covering location problem, set covering location problem, and the maximal covering location problem which were based on time satisfaction and were solved by different algorithms, respectively. Galvao et al. [16], Sheu [17], Rajagopalan et al. [18], 
and Yuan and Wang [19] have researched emergency rescue location problem using a phased manner, respectively. Beraldi and Bruni [20] have researched the phased model by stochastic programming.

Although much research on the emergency rescue location problem has been made with deterministic assumption, the real world is full of nondeterministic factors. There are various forms of uncertainty, such as randomness, fuzziness, and fuzzy randomness [2]. For the uncertainty of emergency events, a large number of scholars have studied how to solve the uncertainty around the emergency location problem. Zhou and Liu [21, 22] proposed facility location-allocation problem with stochastic demands and fuzzy demands. Ren et al. [23] established a two-stage stochastic programming model of the uncertain consumer demand and designed the benders decomposition algorithm. Averbakh and Berman [24] have studied location problem with indefinite requirements by deviations robust optimization methods. Mete and Zabinsky [25] proposed reserves and distribution stochastic optimization method under a variety of possible disasters. Rawls and Turnquist [26] proposed a two-stage stochastic mixed integer programming model in the case of hurricanes and other uncertain disasters. Sheu [27] proposed a dynamic emergency rescue demand management model in the case of insufficient information on large-scale natural disasters. By the definition of trapezoidal fuzzy numbers sort criteria, Guo and Qi [28] gave the emergency material storage location model and algorithm in the fuzzy environment. Ben-Tal et al. [29] processed an emergency rescue location problem under uncertain demand by robust optimization model. In the case of factory supply capacity and customer demand for fuzzy variables, Luo et al. [30] established a distribution center location model with fuzzy variables. Tao and $\mathrm{Hu}$ [31] established a stochastic programming model which is based on set covering for the uncertainty demand and logistics network in emergency relief.

Probability theory can be regarded as a tool to understand objective uncertainty, while sometimes uncertainty shows its subjectivity, known as fuzziness. If the uncertainty behaves neither randomly nor fuzzily, we need a new tool. To describe this type of uncertainty, Liu founded uncertainty theory in 2007 [32] and redefined it in 2010 [33]. Uncertainty theory is a branch of axiomatic mathematics for modeling human uncertainty. Many researchers have made a lot of significant works in this area. Liu [34] proved the linearity of expected value operator. Liu and $\mathrm{Ha}$ [35] derived a formula that can easily calculate the expected values of monotone functions of uncertain variables. Making a great contribution, Liu [36] first proposed uncertain programming which is a type of mathematical programming involving uncertain variables. Many practical problems can be expressed as uncertain programming problems. A number of works in this area have also been developed. Liu and Chen [37] proposed an uncertain multiobjective programming and an uncertain goal programming. Recently, uncertain programming has been extended to the fields of uncertain network optimization [38], Chinese postman problem [39], transportation problem [40], newsboy problem $[41,42]$, and so forth.
In this paper, on the basis of uncertainty theory, the emergency rescue location model is presented and solved. This paper is organized as follows. In Section 2, some basic concepts and properties of uncertainty theory are introduced. In Section 3, the emergency center location model with uncertain rescue transport time and the appropriate decision matrix form are given. In Section 4, a numerical example is presented. The conclusion is given in the last section.

\section{Preliminaries}

In this section, we introduce some foundational concepts and properties on uncertainty theory.

A collection $\mathscr{L}$ of $\Gamma$ is called a $\sigma$-algebra if: (a) $\Gamma \in \mathscr{L}$; (b) if $\Lambda \in \mathscr{L}$, then $\Lambda^{c} \in \mathscr{L}$; (c) if $\Lambda_{1}, \Lambda_{2}, \ldots \in \mathscr{L}$, then $\Lambda_{1} \cup \Lambda_{2} \cup$ $\cdots \in \mathscr{L}$. Each element $\Lambda$ in the $\sigma$-algebra $\mathscr{L}$ is called an event. Uncertain measure is a function from $\mathscr{L}$ to $[0,1]$. In order to present an axiomatic definition of uncertain measure, it is necessary to assign to each event $\Lambda$ a number $\mathscr{M}\{\Lambda\}$ which indicates the belief degree that the event $\Lambda$ will occur. In order to ensure that the number $\mathscr{M}\{\Lambda\}$ has certain mathematical properties, Liu [43] proposed the following three axioms.

Axiom 1 (normality axiom). $\mathscr{M}\{\Gamma\}=1$ for the universal set $\Gamma$.

Axiom 2 (duality axiom). $\mathscr{M}\{\Lambda\}+\mathscr{M}\left\{\Lambda^{c}\right\}=1$ for any event $\Lambda$.

Axiom 3 (subadditivity axiom). For every countable sequence of events $\Lambda_{1}, \Lambda_{2}, \ldots$, we have

$$
\mathscr{M}\left(\bigcup_{i=1}^{\infty} \Lambda_{i}\right) \leq \sum_{i=1}^{\infty} \mathscr{M}\left(\Lambda_{i}\right) .
$$

Axiom 4 (Liu [34], product axiom). Let $\left(\Gamma_{k}, \mathscr{L}_{k}, \mathscr{M}_{k}\right)$ be uncertainty spaces for $k=1,2, \ldots$; the product uncertain measure $\mathscr{M}$ is an uncertain measure satisfying

$$
\mathscr{M}\left(\prod_{k=1}^{\infty} \Lambda_{k}\right)=\bigwedge_{k=1}^{\infty} \mathscr{M}\left(\Lambda_{k}\right)
$$

where $\Lambda_{k}$ are arbitrarily chosen events from $\mathscr{L}_{k}$ for $k=$ $1,2, \ldots$, respectively.

Definition 1 (Liu [43]). An uncertain variable is a measurable function $\xi$ from the uncertainty space $(\Gamma, \mathscr{L}, \mathscr{M})$ to the set of real numbers; that is, for any Borel set $B$ of real numbers, the set

$$
\{\xi \in B\}=\{\gamma \in \Gamma \mid \xi(\gamma) \in B\}
$$

is an event.

Definition 2 (Liu [43]). The uncertainty distribution $\Phi$ of an uncertain variable $\xi$ is defined by

$$
\Phi(x)=\mathscr{M}\{\xi \leq x\}
$$

for any real number $x$. 
Obviously, the uncertainty distribution gives a description of uncertain variables. In many cases, it is sufficient to know the uncertainty distribution rather than the uncertain variable itself. Some useful uncertainty distributions are given here.

Definition 3 (Liu [43]). An uncertain variable $\xi$ is called linear if it has a linear uncertainty distribution

$$
\Phi(x)= \begin{cases}0, & \text { if } x \leq a, \\ \frac{x-a}{b-a}, & \text { if } a \leq x \leq b, \\ 1, & \text { if } x \geq b\end{cases}
$$

denoted by $\mathscr{L}(a, b)$, where $a$ and $b$ are real numbers with $a<$ $b$.

To calculate the uncertain measure from an uncertainty distribution, we introduce relevant theorems and definitions.

Theorem 4 (Liu [33], measure inversion theorem). Let $\xi$ be an uncertain variable with continuous uncertainty distribution $\Phi$; then, for any real number $x$, one has

$$
\mathscr{M}\{\xi \leq x\}=\Phi(x), \quad \mathscr{M}\{\xi \geq x\}=1-\Phi(x) .
$$

Definition 5 (Liu [43]). An uncertainty distribution $\Phi$ is said to be regular if its inverse function $\Phi^{-1}(\alpha)$ exists and is unique for each $a \in(0,1)$.

Definition 6 (Liu [43], inverse uncertainty distribution). Let $\xi$ be an uncertain variable with regular uncertainty distribution $\Phi$; then the inverse function $\Phi^{-1}$ is called the inverse uncertainty distribution of $\xi$.

Now, we give some useful inverse uncertainty distributions.

Definition 7 (Liu [43]). The inverse uncertainty distribution of linear uncertain variable $\mathscr{L}(a, b)$ is

$$
\Phi^{-1}(\alpha)=(1-\alpha) a-\alpha b .
$$

Theorem 8 (Liu [33]). Let $\xi_{1}, \xi_{2}, \ldots, \xi_{n}$ be independent variables with regular uncertainty distributions $\Phi_{1}, \Phi_{2}, \ldots, \Phi_{n}$, respectively. If $f$ is a strictly increasing function, then

$$
\xi=f\left(\xi_{1}+\xi_{2}+\cdots+\xi_{n}\right)
$$

is an uncertain variable with inverse uncertainty distribution

$$
\Psi^{-1}(\alpha)=f\left(\Phi_{1}^{-1}(\alpha), \Phi_{2}^{-1}(\alpha), \ldots, \Phi_{n}^{-1}(\alpha)\right) .
$$

Definition 9 (Liu [43]). Let $\xi_{1}, \xi_{2}, \ldots, \xi_{n}$ be independent variables with regular uncertainty distributions $\Phi_{1}, \Phi_{2}, \ldots, \Phi_{n}$, respectively. Since

$$
f\left(x_{1}, x_{2}, \ldots, x_{n}\right)=x_{1} \wedge x_{2} \wedge \cdots \wedge x_{n}
$$

is a strictly increasing function, the minimum

$$
\xi=\xi_{1} \wedge \xi_{2} \wedge \cdots \wedge \xi_{n}
$$

is an uncertain variable with inverse uncertainty distribution

$$
\Psi^{-1}(\alpha)=\Phi_{1}^{-1}(\alpha) \wedge \Phi_{2}^{-1}(\alpha) \wedge \cdots \wedge \Phi_{n}^{-1}(\alpha) .
$$

Expected value is the average value of uncertain variable in the sense of uncertain measure and represents the size of uncertain variable.

Theorem 10 (Liu [43]). Let $\xi$ be an uncertain variable with regular uncertainty distribution $\Phi$; if the expected value exists, then

$$
E[\xi]=\int_{0}^{1} \Phi^{-1}(\alpha) d \alpha
$$

Definition 11 (Liu [43]). Let $\xi \sim \mathscr{L}(a, b)$ be a linear uncertain variable. Then, its inverse uncertainty distribution is $\Phi^{-1}(\alpha)=(1-\alpha) a-\alpha b$, and its expected value is

$$
E[\xi]=\int_{0}^{1} \Phi^{-1}(\alpha) d \alpha=\int_{0}^{1}((1-\alpha) a+\alpha b) d \alpha=\frac{a+b}{2}
$$

Theorem 12 (Liu [33]). Let $\xi$ and $\eta$ be independent uncertain variables with finite expected values. Then, for any real numbers $a$ and $b$, one has

$$
E[a \xi+b \eta]=a E[\xi]+b E[\eta] .
$$

\section{Problem Descriptions}

Here, we assume that there is a group of rescue demand points. Each has its location and population. In order to supply the demand points, we will construct a group of emergency rescue centers, respectively, within given locations. A single rescue demand point can be supplied by multiple emergency rescue centers and a single emergency rescue center can supply multiple rescue demand points. In order to solve this problem, we list model parameters and decision variables as follows.

\subsection{Index Set}

$i$ is index of emergency rescue center.

$j$ is index of rescue demand point.

\subsection{Model Parameters}

$C_{i}$ is the construction cost of emergency rescue center $(i=1,2, \ldots, m)$.

$c_{i j}$ is the per hour transport cost between emergency rescue center $i$ and rescue demand point $j(i=$ $1,2, \ldots, m ; j=1,2, \ldots, n)$.

$\xi_{i j}$ is the uncertain time between emergency rescue center $i$ and rescue demand point $j(i=1,2, \ldots, m ; j=$ $1,2, \ldots, n)$.

$\Phi_{i j}$ is the uncertainty distribution of $\xi_{i j}(i=1,2, \ldots, m$; $j=1,2, \ldots, n)$. 
$\Phi_{i j}^{-1}$ is the inverse uncertainty distribution of $\xi_{i j}(i=$ $1,2, \ldots, m ; j=1,2, \ldots, n)$.

$d_{i j}$ is the distance between emergency rescue center $i$ and rescue demand point $j(i=1,2, \ldots, m ; j=$ $1,2, \ldots, n)$.

$T_{j}$ is the time limit of rescue demand point $j(j=$ $1,2, \ldots, n)$.

$p$ is the maximum number of emergency rescue center.

$p_{i}$ is the maximum number of rescue demand point covered by each emergency rescue center $(i=$ $1,2, \ldots, m)$.

$\alpha$ is confidence level.

\subsection{Decision Variables. Consider}

$x_{i}= \begin{cases}1, & \text { if emergency rescue center } i \text { is selected, } \\ 0, & \text { otherwise, }\end{cases}$ $y_{i j}= \begin{cases}1, & \begin{array}{r}\text { if rescue demand point } j \\ \text { be serviced by emergency rescue point } i,\end{array} \\ 0, & \text { otherwise. }\end{cases}$

3.4. Uncertain Model of Emergency Rescue Location Problem. $C$ is the total cost that includes the construction cost and transportation cost, and then

$$
C=\sum_{i=1}^{m} C_{i} x_{i}+\sum_{j=1}^{n} \sum_{i=1}^{m} c_{i j} \xi_{i j} y_{i j}
$$

In decision making, we hope to minimize the total cost. By the definition of $\xi_{i j}$, the expectation of the total cost is

$$
C(x, y, \xi)=E\left[\sum_{i=1}^{m} C_{i} x_{i}+\sum_{j=1}^{n} \sum_{i=1}^{m} c_{i j} \xi_{i j} y_{i j}\right]
$$

Thus, the objective function of the decision problem is to make the expected value of the total cost minimum; that is

$$
\min C(x, y, \xi)=E\left[\sum_{i=1}^{m} C_{i} x_{i}+\sum_{j=1}^{n} \sum_{i=1}^{m} c_{i j} \xi_{i j} y_{i j}\right]
$$

Consider the time urgency of emergency rescue center location, that is, in a limited period of time, to complete the emergency work after the disaster. Hence, for each rescue demand point, the shortest time of the emergency rescue center to the rescue demand point should be in a predetermined time. Then, we give the constraint of time:

$$
\bigwedge_{i=1}^{m} \xi_{i j} y_{i j} \leq T_{j} \quad(j=1,2, \ldots, n)
$$

In the actual calculation, the affected point $j$ might not served by the rescue center $i$, then $y_{i j}=0$, and $\wedge_{i=1}^{m} \xi_{i j} y_{i j}=0$.
In order to avoid such a result, we define the transportation time as

$$
K_{i j}\left(\xi_{i j}, y_{i j}\right)= \begin{cases}\xi_{i j} y_{i j}, & \text { if } y_{i j}=1 \\ Q, & \text { if } y_{i j}=0\end{cases}
$$

where $Q$ is a very large number.

And an uncertain time constraint is given:

$$
\mathscr{M}\left(\bigwedge_{i=1}^{m} K_{i j}\left(\xi_{i j}, y_{i j}\right) \leq T_{j}\right) \geq \alpha_{j} .
$$

Based on the analysis of the decision making process, the emergency rescue location problem model is stated as follows. Consider

$$
\begin{array}{ll}
\min & C(x, y, \xi)=E\left[\sum_{i=1}^{m} C_{i} x_{i}+\sum_{j=1}^{n} \sum_{i=1}^{m} c_{i j} \xi_{i j} y_{i j}\right] \\
\text { s.t. } \quad & M\left(\bigwedge_{i=1}^{m} K_{i j}\left(\xi_{i j}, y_{i j}\right) \leq T_{j}\right) \geq \alpha_{j} \\
& \sum_{i=1}^{m} x_{i} \leq p \\
& y_{i j} \leq x_{i} \\
& \sum_{j=1}^{n} y_{i j} \leq p_{i} \quad(i=1,2, \ldots, m) \\
& x_{i}, y_{i j} \in\{0,1\} .
\end{array}
$$

Objective (23) means the minimization of total construction cost of emergency rescue center. Constraint (24) means the time limit. Constraints (25) and (26) mean that emergency rescue center to participate in the rescue work does not exceed the number of $p$. Constraint (27) means the number of restrictions of emergency rescue center to supply rescue demand points.

Then, this programming problem can be transformed into an equivalent deterministic programming problem by uncertainty theory as follows. Consider

$$
\begin{array}{ll}
\min & C=\sum_{i=1}^{m} C_{i} x_{i}+\sum_{j=1}^{n} \sum_{i=1}^{m} c_{i j}\left(\int_{0}^{1} \Phi_{i j}^{-1}(\alpha) d \alpha\right) y_{i j} \\
\text { s.t. } & \bigwedge_{i=1}^{m} \Phi_{i j}^{-1}\left(\alpha_{j}, y_{i j}\right) \leq T_{j} \\
& \sum_{i=1}^{m} x_{i} \leq p \\
& y_{i j} \leq x_{i} \\
& \sum_{j=1}^{n} y_{i j} \leq p_{i} \quad(i=1,2, \ldots, m) \\
& x_{i}, y_{i j} \in\{0,1\} .
\end{array}
$$


TABle 1: Distance (unit: km).

\begin{tabular}{lccccc}
\hline Rescue demand point & $\mathrm{x} 1$ & $\mathrm{x} 2$ & $\mathrm{x} 3$ & $\mathrm{x} 4$ & $\mathrm{x} 5$ \\
\hline 1 & 13.7 & 5.2 & 9.3 & 12.0 & 7.2 \\
2 & 19.0 & 13.1 & 8.4 & 3.2 & 7.6 \\
3 & 12.0 & 3.3 & 4.9 & 8.0 & 4.3 \\
4 & 14.5 & 8.0 & 3.6 & 2.5 & 3.8 \\
5 & 5.8 & 3.4 & 3.8 & 12.7 & 11.2 \\
6 & 6.7 & 4.3 & 4.1 & 19.3 & 9.8 \\
7 & 11.7 & 9.2 & 3.8 & 5.6 & 9.2 \\
8 & 6.0 & 7.9 & 6.3 & 10.9 & 12.6 \\
9 & 21.0 & 15.0 & 12.3 & 11.2 & 8.5 \\
10 & 13.0 & 5.2 & 8.4 & 10.0 & 21.0 \\
11 & 3.5 & 8.6 & 5.3 & 6.2 & 7.5 \\
12 & 20.0 & 16.5 & 13.4 & 12.1 & 5.7 \\
13 & 11.2 & 3.8 & 5.7 & 9.8 & 8.4 \\
14 & 14.3 & 8.9 & 7.5 & 12.5 & 10.0 \\
15 & 2.5 & 3.8 & 1.8 & 5.9 & 9.8 \\
16 & 15.6 & 5.6 & 4.8 & 12.3 & 21.0 \\
17 & 3.2 & 4.3 & 18.6 & 9.7 & 8.5 \\
18 & 7.8 & 19.3 & 8.5 & 3.2 & 12.8 \\
19 & 4.9 & 12.9 & 8.9 & 13.5 & 21.0 \\
20 & 15.6 & 6.5 & 13.8 & 4.2 & 10.0 \\
\hline
\end{tabular}

\section{The Numerical Example}

After our model is designed and a conceptual method is found, a realistic problem is how it works when sample data are given. An example of a group of rescue demand points and planned locations for construction of emergency rescue center is presented in the following tables. The distance between those locations and the construction cost and transport cost as well as efficiency restrictions are also given (there are 20 rescue demand points and 5 alternative emergency rescue centers). For simplicity, the transport time is performed as a linear uncertain variable, that is, $\xi_{i j} \sim \mathscr{L}(a, b)$; then we give experts empirical data table (Tables 1, 2, and 3). The location program requires the following parameters $p=3, p_{1}=$ $p_{2}=p_{3}=p_{4}=p_{5}=15$. When a solution, location selection vector, and requirement cover matrix are given, we can calculate the objective value. It is also clear to determine whether the solution is reasonable and whether it can meet every constraint in our model.

We use the down mountain algorithm to solve the numerical example. The algorithm we actually performed is to search for a reasonable and the most efficient solution. Obviously, the constrains can be used to reduce the scope so that the best solution can quickly be found.

We get the optimal requirement cover matrix by calculation (Table 4). The best solution shows that the emergency rescue location selection vector is $(0,1,1,0,1)$; that is, the emergency rescue centers $\mathrm{x} 2, \mathrm{x} 3$, and $\mathrm{x} 5$ are selected. And the emergency rescue center $\mathrm{x} 2$ provides services for the rescue demand points numbers $1,4,5,7,8,10,11,12,13,14,15,16$, $17,18,19$, and 20 . The emergency rescue center $\mathrm{x} 3$ provides services for the rescue demand points numbers $2,3,4,5,6$,
TABLE 2: Construction cost and transport cost.

\begin{tabular}{llllll}
\hline $\begin{array}{l}\text { Per hour transport } \\
\text { cost/million }\end{array}$ & $\mathrm{x} 1$ & $\mathrm{x} 2$ & $\mathrm{x} 3$ & $\mathrm{x} 4$ & $\mathrm{x} 5$ \\
\hline 1 & 2.1 & 2.2 & 2.3 & 2.0 & 2.0 \\
2 & 2.3 & 1.8 & 2.5 & 1.7 & 1.5 \\
3 & 19 & 1.9 & 1.6 & 2.4 & 3.0 \\
4 & 1.8 & 1.5 & 1.9 & 2.1 & 2.1 \\
5 & 1.7 & 2.5 & 1.5 & 2.3 & 3.0 \\
6 & 2.1 & 1.7 & 2.5 & 2.2 & 2.4 \\
7 & 2.3 & 1.6 & 2.1 & 2.4 & 2.1 \\
8 & 2.4 & 1.4 & 1.8 & 2.1 & 2.0 \\
9 & 2.5 & 2.0 & 1.7 & 2.5 & 2.0 \\
10 & 3.0 & 2.3 & 1.2 & 2.6 & 2.1 \\
11 & 1.8 & 1.3 & 1.3 & 3.0 & 2.6 \\
12 & 1.4 & 1.8 & 2.0 & 1.3 & 2.5 \\
13 & 2.0 & 1.6 & 2.1 & 1.8 & 2.8 \\
14 & 1.8 & 1.9 & 2.5 & 1.9 & 1.2 \\
15 & 1.6 & 2.1 & 2.4 & 1.5 & 1.6 \\
16 & 2.1 & 2.3 & 1.2 & 1.4 & 1.8 \\
17 & 2.6 & 1.1 & 2.3 & 2.6 & 1.7 \\
18 & 2.2 & 0.9 & 2.2 & 2.5 & 3.0 \\
19 & 1.6 & 1.6 & 2.2 & 2.2 & 1.9 \\
20 & 1.8 & 1.7 & 1.8 & 2.1 & 2.2 \\
\hline Construction cost of & 98 & 97 & 100 & 96 & 99 \\
rescue center/million & & & & & \\
\hline
\end{tabular}

$7,8,10,13,15,16,18,19$, and 20 . The emergency rescue center $\mathrm{x} 5$ provides services for the rescue demand points numbers $1,2,3,4,7,9,10,11,12,14$, and 17 . Then, the objective value is 672.295 .

\section{Conclusions}

Emergency rescue location problem is an important issue in emergency management. An emergency rescue location model under uncertain environment is presented in this paper. An uncertain variable is introduced to describe the transport time between emergency rescue centers and rescue demand points. Based on uncertain theory, an uncertain expected cost minimization model was proposed. Finally, a numerical example has been presented to illustrate the effectiveness of the model.

There are some suggestions for future research. We could introduce additional uncertain parameters into this model or construct a dynamic uncertain model, so that it should be more suitable in emergency management.

\section{Conflict of Interests}

The author declares that there is no conflict of interests regarding the publication of this paper. 
TABLE 3: Linear uncertain distribution of transport time (unit: hour).

\begin{tabular}{|c|c|c|c|c|c|c|}
\hline Rescue demand point & $\mathrm{x} 1$ & $\mathrm{x} 2$ & $\mathrm{x} 3$ & $\mathrm{x} 4$ & $\mathrm{x} 5$ & Time limit \\
\hline 1 & $\mathscr{L}(7.0,9.1)$ & $\mathscr{L}(5.3,7.9)$ & $\mathscr{L}(5.6,8.4)$ & $\mathscr{L}(6.9,10.3)$ & $\mathscr{L}(2.1,3.2)$ & 6 \\
\hline 2 & $\mathscr{L}(8.2,13.5)$ & $\mathscr{L}(8.1,12.2)$ & $\mathscr{L}(4.6,6.9)$ & $\mathscr{L}(1.3,2.5)$ & $\mathscr{L}(2.5,3.8)$ & 6 \\
\hline 3 & $\mathscr{L}(6.5,8.6)$ & $\mathscr{L}(1.4,2.2)$ & $\mathscr{L}(1.5,2.3)$ & $\mathscr{L}(7.0,11)$ & $\mathscr{L}(1.3,2.0)$ & 3 \\
\hline 4 & $\mathscr{L}(7.5,9.5)$ & $\mathscr{L}(3.0,4.5)$ & $\mathscr{L}(1.3,3.0)$ & $\mathscr{L}(1.0,2.0)$ & $\mathscr{L}(1.0,1.1)$ & 3 \\
\hline 5 & $\mathscr{L}(2.5,4.5)$ & $\mathscr{L}(1.0,1.6)$ & $\mathscr{L}(1.2,2.3)$ & $\mathscr{L}(7.1,9.1)$ & $\mathscr{L}(4.2,6.2)$ & 4 \\
\hline 6 & $\mathscr{L}(3.0,5.0)$ & $\mathscr{L}(1.2,2.3)$ & $\mathscr{L}(1.5,2.6)$ & $\mathscr{L}(8.1,13.0)$ & $\mathscr{L}(3.1,4.7)$ & 4 \\
\hline 7 & $\mathscr{L}(6.2,8.4)$ & $\mathscr{L}(3.9,5.9)$ & $\mathscr{L}(1.5,3.0)$ & $\mathscr{L}(2.3,4.3)$ & $\mathscr{L}(3.0,4.6)$ & 5 \\
\hline 8 & $\mathscr{L}(2.9,4.8)$ & $\mathscr{L}(3.3,6.0)$ & $\mathscr{L}(3.8,5.8)$ & $\mathscr{L}(3.5,5.5)$ & $\mathscr{L}(4.6,6.9)$ & 5 \\
\hline 9 & $\mathscr{L}(6.0,8.1)$ & $\mathscr{L}(5.5,8.0)$ & $\mathscr{L}(5.0,8.0)$ & $\mathscr{L}(6.3,11.3)$ & $\mathscr{L}(2.5,3.8)$ & 6 \\
\hline 10 & $\mathscr{L}(8.0,12.0)$ & $\mathscr{L}(4.7,8.0)$ & $\mathscr{L}(4.6,9.6)$ & $\mathscr{L}(6.8,10.8)$ & $\mathscr{L}(3.5,6.8)$ & 7 \\
\hline 11 & $\mathscr{L}(7.0,18.0)$ & $\mathscr{L}(5.6,9.0)$ & $\mathscr{L}(8.9,12.6)$ & $\mathscr{L}(7.9,10.8)$ & $\mathscr{L}(3.7,7.0)$ & 7 \\
\hline 12 & $\mathscr{L}(10.8,13.8)$ & $\mathscr{L}(4.7,9.0)$ & $\mathscr{L}(7.9,10.6)$ & $\mathscr{L}(6.5,10.8)$ & $\mathscr{L}(2.0,4.0)$ & 8 \\
\hline 13 & $\mathscr{L}(10.8,13.8)$ & $\mathscr{L}(3.4,7.9)$ & $\mathscr{L}(5.8,10.6)$ & $\mathscr{L}(6.5,11.8)$ & $\mathscr{L}(6.0,10.0)$ & 7 \\
\hline 14 & $\mathscr{L}(9.0,13.0)$ & $\mathscr{L}(5.0,8.9)$ & $\mathscr{L}(5.0,9.0)$ & $\mathscr{L}(6.5,12.0)$ & $\mathscr{L}(5.4,9.8)$ & 8 \\
\hline 15 & $\mathscr{L}(2.0,5.0)$ & $\mathscr{L}(2.5,5.5)$ & $\mathscr{L}(1.2,3.0)$ & $\mathscr{L}(5.0,8.0)$ & $\mathscr{L}(5.4,9.8)$ & 4 \\
\hline 16 & $\mathscr{L}(8.0,11.3)$ & $\mathscr{L}(2.5,6.0)$ & $\mathscr{L}(2.3,5.6)$ & $\mathscr{L}(6.8,11.2)$ & $\mathscr{L}(10.2,13.5)$ & 7 \\
\hline 17 & $\mathscr{L}(2.1,4.2)$ & $\mathscr{L}(3.5,6.0)$ & $\mathscr{L}(8.9,12.5)$ & $\mathscr{L}(7.5,11.0)$ & $\mathscr{L}(7.0,10.0)$ & 6 \\
\hline 18 & $\mathscr{L}(6.5,10.5)$ & $\mathscr{L}(12.5,14.5)$ & $\mathscr{L}(6.5,11.5)$ & $\mathscr{L}(2.0,4.0)$ & $\mathscr{L}(7.0,11.0)$ & 8 \\
\hline 19 & $\mathscr{L}(3.5,6.0)$ & $\mathscr{L}(10.5,13.5)$ & $\mathscr{L}(6.5,11.5)$ & $\mathscr{L}(9.0,14.0)$ & $\mathscr{L}(14.0,19.5)$ & 8 \\
\hline 20 & $\mathscr{L}(9.5,13.5)$ & $\mathscr{L}(4.5,10.6)$ & $\mathscr{L}(9.5,11.5)$ & $\mathscr{L}(3.0,4.6)$ & $\mathscr{L}(7.0,11.0)$ & 7 \\
\hline
\end{tabular}

TABLE 4: Requirement cover solution.

\begin{tabular}{lcccccccccccccccccccccccccc}
\hline $\begin{array}{l}\text { Emergency rescue } \\
\text { center }\end{array}$ & 1 & 2 & 3 & 4 & 5 & 6 & 7 & 8 & 9 & 10 & 11 & 12 & 13 & 14 & 15 & 16 & 17 & 18 & 19 & 20 \\
\hline $\mathrm{x} 1$ & $\times$ & $\times$ & $\times$ & $\times$ & $\times$ & $\times$ & $\times$ & $\times$ & $\times$ & $\times$ & $\times$ & $\times$ & $\times$ & $\times$ & $\times$ & $\times$ & $\times$ & $\times$ & $\times$ & $\times$ \\
$\mathrm{x} 2$ & 1 & 0 & 0 & 0 & 1 & 1 & 0 & 1 & 1 & 0 & 1 & 1 & 1 & 1 & 1 & 1 & 1 & 1 & 1 & 1 \\
$\mathrm{x} 3$ & 0 & 1 & 1 & 1 & 1 & 1 & 1 & 1 & 0 & 1 & 0 & 0 & 1 & 0 & 1 & 1 & 0 & 1 & 1 & 1 \\
x4 & $\times$ & $\times$ & $\times$ & $\times$ & $\times$ & $\times$ & $\times$ & $\times$ & $\times$ & $\times$ & $\times$ & $\times$ & $\times$ & $\times$ & $\times$ & $\times$ & $\times$ & $\times$ & $\times$ & $\times$ \\
$\mathrm{x} 5$ & 1 & 1 & 1 & 1 & 0 & 0 & 1 & 0 & 1 & 1 & 1 & 1 & 0 & 1 & 0 & 0 & 1 & 0 & 0 & 0 \\
\hline
\end{tabular}

\section{Acknowledgments}

The author would like to thank the editors and reviewers for their truly helpful comments and suggestions which led to a very improved presentation. And this work is supported by the Fundamental Research Funds for the Central Universities, the Special Fund of Civil Aviation University of China (Grant no. 3122014D034), and Research Foundation of Civil Aviation University of China (Grant no. 05yk33s).

\section{References}

[1] X. Chen and C. X. Wang, "Optimization model of location for emergency relief centers under fuzzy random demand," Operations Research and Management Science, vol. 21, no. 5, pp. 73-77, 2012.

[2] J. Dai and X. J. Guan, "Literature review on robust optimization of location allocation of mass emergency rescue resources distribution nodes," Logistics Technology, vol. 33, no. 1, pp. 8-11, 2014.

[3] L. Cooper, "Location-allocation problems," Operations Research, vol. 11, pp. 331-343, 1963.
[4] R. Roth, "Computer solution to minimum cover problem," Operational Research, vol. 17, no. 3, pp. 455-465, 1969.

[5] C. Toregas and R. Swaim, "The location of emergency service facilities," Operational Research, vol. 19, no. 6, pp. 1363-1373, 1971.

[6] P. Murali, F. Ordóñez, and M. M. Dessouky, "Facility location under demand uncertainty: response to a large-scale bio-terror attack," Socio-Economic Planning Sciences, vol. 46, no. 1, pp. 7887, 2012.

[7] B. A. Murtagh and S. R. Niwattisyawong, "Efficient method for the multi-depot location-allocation problem," Journal of the Operational Research Society, vol. 33, no. 7, pp. 629-634, 1982.

[8] S. I. Harewood, "Emergency ambulance deployment in Barbados: a multi-objective approach," Journal of the Operational Research Society, vol. 53, no. 2, pp. 185-192, 2002.

[9] L. Brotcorne, G. Laporte, and F. Semet, "Ambulance location and relocation models," European Journal of Operational Research, vol. 147, no. 3, pp. 451-463, 2003.

[10] J. B. Goldberg, "Operations research models for the deployment of emergency services vehicles," EMS Management Journal, vol. 1, no. 1, pp. 20-39, 2004. 
[11] O. I. Alsalloum and G. K. Rand, "Extensions to emergency vehicle location models," Computers and Operations Research, vol. 33, no. 9, pp. 2725-2743, 2006.

[12] F. Tovia, "An emergency logistics response system for natural disasters," International Journal of Logistics:Research and Applications, vol. 10, no. 3, pp. 173-186, 2007.

[13] L. Fang and J. M. He, "Optimal location model of emergency systems by a given deadline," Journal of Industrial Engineering and Engineering Management, vol. 18, no. 1, pp. 48-51, 2004.

[14] D.-W. Wang and G.-X. Zhang, "Model and algorithm to optimize location of catastrophic rescue center," Journal of Northeastern University, vol. 26, no. 10, pp. 953-956, 2005.

[15] F. Y. Ma, Y. Liu, and C. Yang, "Time-satisfaction-based set covering location problem and applications of greedy algorithms," Journal of Wuhan University of Science and Technology, vol. 29, no. 6, pp. 631-635, 2006.

[16] R. D. Galvao, F. Y. Chiyoshi, and R. Morabito, "Towards unified formulations and extensions of two classical probabilistic location models," Computers \& Operations Research, vol. 32, no. 1, pp. 15-33, 2005.

[17] J. B. Sheu, "An emergency logistics distribution approach for quick response to urgent relief demand in disasters," Transportation Research Part E: Logistics and Transportation Review, vol. 43, no. 6, pp. 687-709, 2007.

[18] H. K. Rajagopalan, C. Saydam, and J. Xiao, "A multiperiod set covering location model for dynamic redeployment of ambulances," Computers \& Operations Research, vol. 35, no. 3, pp. 814-826, 2008.

[19] Y. Yuan and D. Wang, "Path selection model and algorithm for emergency logistics management," Computers and Industrial Engineering, vol. 56, no. 3, pp. 1081-1094, 2009.

[20] P. Beraldi and M. E. Bruni, "A probabilistic model applied to emergency service vehicle location," European Journal of Operational Research, vol. 196, no. 1, pp. 323-331, 2009.

[21] J. Zhou and B. Liu, "New stochastic models for capacitated location-allocation problem," Computers and Industrial Engineering, vol. 45, no. 1, pp. 111-125, 2003.

[22] J. Zhou and B. Liu, "Modeling capacitated location-allocation problem with fuzzy demands," Computers and Industrial Engineering, vol. 53, no. 3, pp. 454-468, 2007.

[23] M. M. Ren, C. Yang, and B. He, "An intergrated optimal approach for facility location and its size decision with uncertain demand," Systems Engineering, vol. 25, no. 6, pp. 1-5, 2007.

[24] I. Averbakh and O. Berman, "Minmax regret median location on a network under uncertainty," Informs Journal on Computing, vol. 12, no. 2, pp. 104-110, 2000.

[25] H. O. Mete and Z. B. Zabinsky, "Stochastic optimization of medical supply location and distribution in disaster management," International Journal of Production Economics, vol. 126, no. 1, pp. 76-84, 2010.

[26] C. G. Rawls and M. A. Turnquist, "Pre-positioning of emergency supplies for disaster response," Transportation Research B: Methodological, vol. 44, no. 4, pp. 521-534, 2010.

[27] J. B. Sheu, "Dynamic relief-demand management for emergency logistics operations under large-scale disasters," Transportation Research Part E: Logistics and Transportation Review, vol. 46, no. 1, pp. 1-17, 2010.

[28] Z. X. Guo and M. R. Qi, "Location model and its algorithm for emergency material storage under fuzzy environment," Computer Engineering and Applications, vol. 46, no. 25, pp. 214216, 2010 .
[29] A. Ben-Tal, B. D. Chung, S. R. Mandala, and T. Yao, "Robust optimization for emergency logistics planning: risk mitigation in humanitarian relief supply chains," Transportation Research Part B: Methodological, vol. 45, no. 8, pp. 1177-1189, 2011.

[30] H. X. Luo, Z. Z. Yuan, and S. S. Peng, "Distribution center location with uncertain supply and demand," Science Technology and Engineering, vol. 12, no. 30, pp. 8100-8102, 2012.

[31] S. Tao and Z. H. Hu, "Facility location in emergency relief with uncertain demand and logistics network," Journal of Computer Applications, vol. 32, no. 9, pp. 2534-2537, 2012.

[32] B. Liu, Uncertainty Theory, Springer, Berlin, Germany, 2nd edition, 2007.

[33] B. Liu, Uncertainty Theory: A Branch of Mathmatics for Model Human Uncertainty, Springer, Berlin, Germany, 2010.

[34] B. Liu, "Some research problems in uncertainty theory," Journal of Uncertain Systems, vol. 3, no. 1, pp. 3-10, 2009.

[35] Y. H. Liu and M. H. Ha, "Expected value of function of uncertain variables," Journal of Uncertain Systems, vol. 4, no. 3, pp. 181-186, 2010.

[36] B. Liu, Theory and Practice of Uncertain Programming, Springer, Berlin, Germany, 2nd edition, 2009.

[37] B. Liu and X. W. Chen, "Uncertain multiobjective programming and uncertain goal programming," Tech. Rep., 2012.

[38] Y. Gao, "Uncertain models for single facility location problems on networks," Applied Mathematical Modelling, vol. 36, no. 6, pp. 2592-2599, 2012.

[39] B. Zhang and J. Peng, "Uncertain programming model for Chinese postman problem with uncertain weights," Industrial Engineering and Management Systems, vol. 11, no. 1, pp. 18-25, 2012.

[40] Y. H. Sheng and K. Yao, "Fixed charge transportation problem in uncertain environment," Industrial Engineering and Management Systems, vol. 11, no. 2, pp. 183-187, 2012.

[41] S. B. Ding, "Uncertain random newsboy problem," Journal of Intelligent \& Fuzzy Systems, vol. 26, no. 1, pp. 483-490, 2014.

[42] S. B. Ding, "Uncertain multi-product newsboy problem with chance constraint," Applied Mathematics and Computation, vol. 223, pp. 139-146, 2013.

[43] B. Liu, Uncertainty Theory, Uncertainty Theory Laboratory, 4th edition, 2013. 


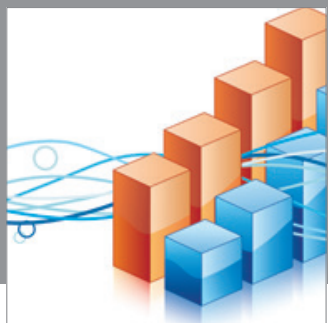

Advances in

Operations Research

mansans

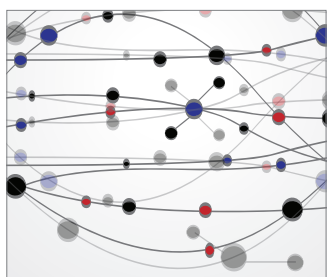

The Scientific World Journal
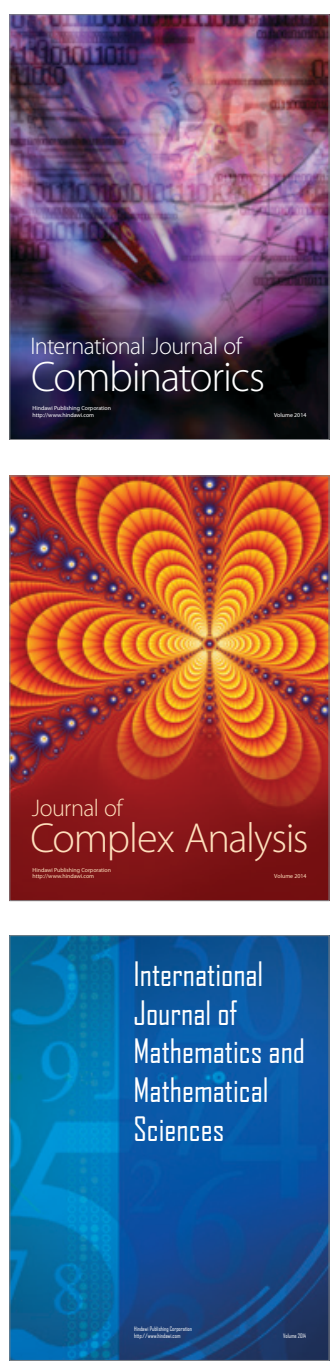
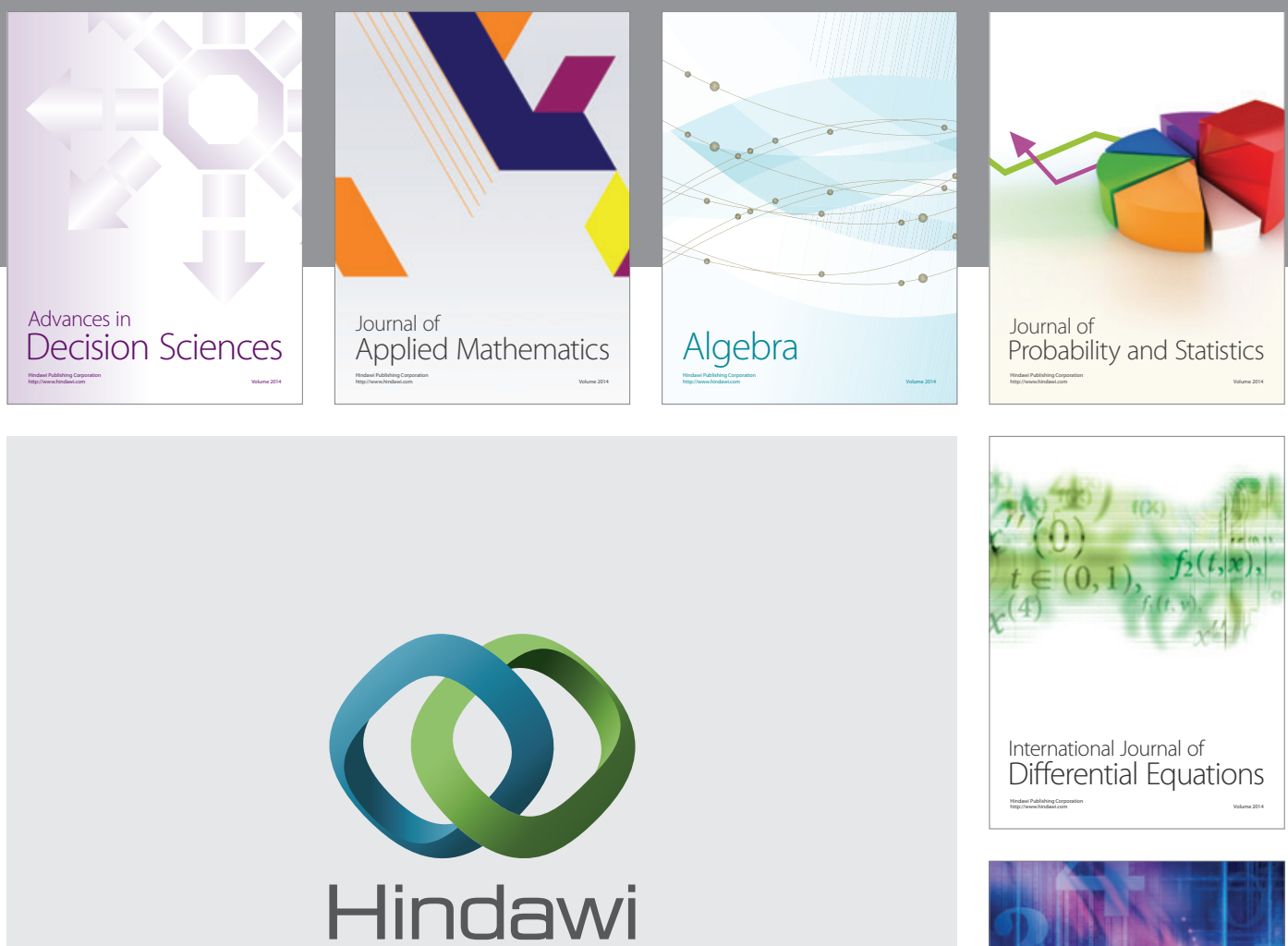

Submit your manuscripts at http://www.hindawi.com


Journal of

Function Spaces

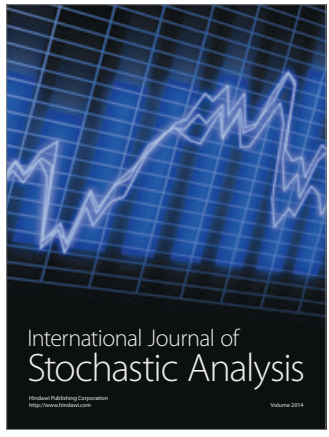

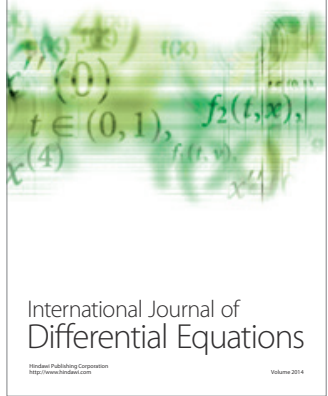
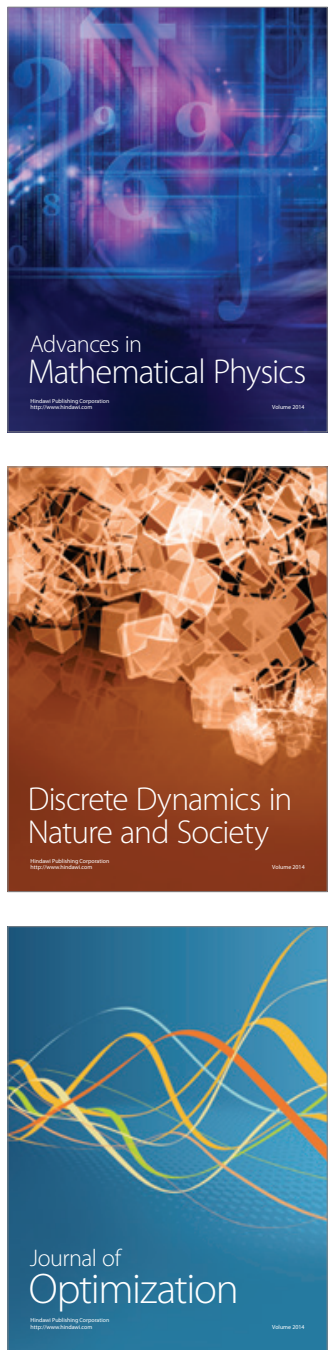